\title{
Crystal structure of 1-(benzyloxy)urea, $\mathrm{C}_{8} \mathrm{H}_{10} \mathrm{~N}_{2} \mathrm{O}_{2}$
}

\author{
Xi Mai ${ }^{\mathrm{IIII}}$, Hong-Ying Xia ${ }^{\mathrm{II}}$, Yu-Sheng Cao ${ }^{*}, \mathrm{I}$, Xiao-San $\mathrm{Lu}^{\mathrm{II}}$ and Yi-Jing Liao ${ }^{\mathrm{II}}$ \\ I Nanchang University, Sino-German Joint Research Institute, Nanchang 330047, P. R. China \\ II Nanchang University, Medical College, Department of Pharmacy, Nanchang 330006, P. R. China
}

Received June 19, 2009, accepted and available on-line August 24, 2009; CCDC no. 1267/2682

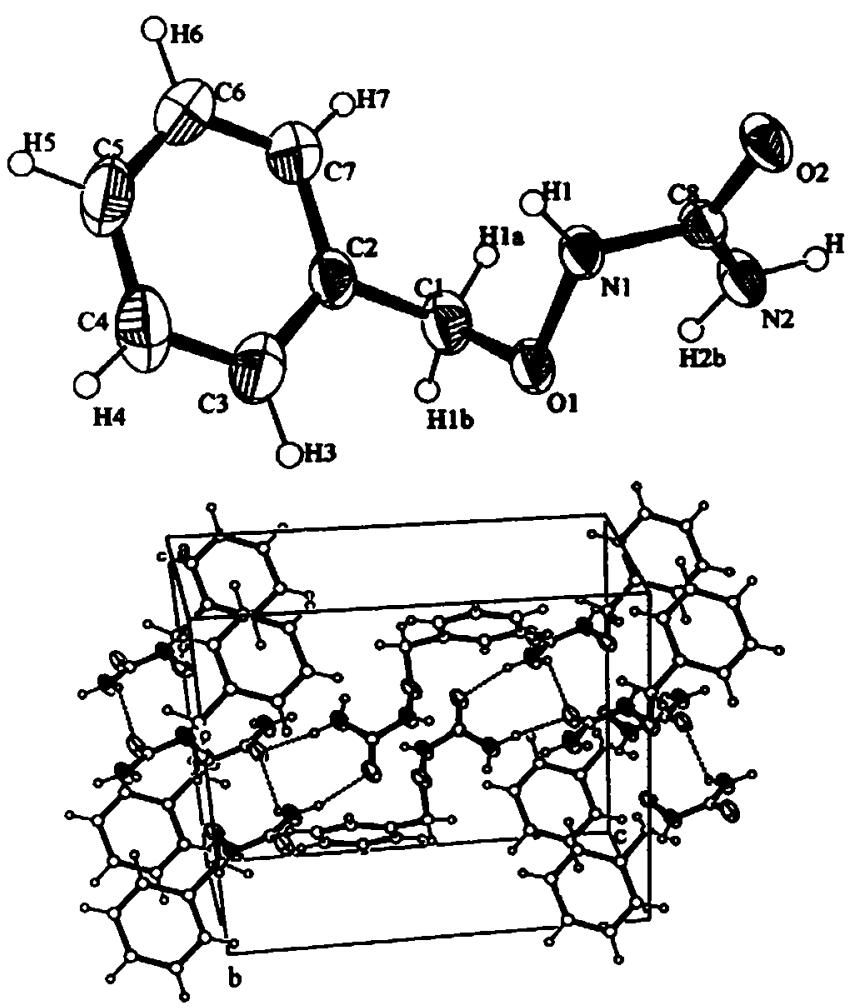

Abstract

$\mathrm{C}_{8} \mathrm{H}_{10} \mathrm{~N}_{2} \mathrm{O}_{2}$, monoclinic, $P 121 / c 1$ (no. 14), $a=12.456(3) \AA$, $b=5.008(1) \AA, c=13.681(4) \AA, \beta=96.017(4)^{\circ}, V=848.7 \AA^{3}$, $Z=4, R_{g r}(F)=0.057, w R_{\text {reff }}\left(F^{2}\right)=0.166, T=296 \mathrm{~K}$.

\section{Source of material}

The title compound was synthesized by reaction of hydroxyurea ( $0.026 \mathrm{~mol})$ with benzyl chloride $(0.034 \mathrm{~mol})$ in methanol $(100$ $\mathrm{ml})$ in the presence of potassium hydroxide $(0.034 \mathrm{~mol})$. After refluxing for $13 \mathrm{~h}$, the solution was distilled in the reduced pressure at $35^{\circ} \mathrm{C}$. The resulting crude solid was filtered and washed by trichloromethane, then recrystallized in acetone/trichloromethane $(5: 2, v / v)$, filtered and dried. Colorless needle-shaped single crystals of the title compound suitable for $\mathrm{X}$-ray structure determination were obtained by slow evaporation of the mixed solvent acetone $/ n$-hexane $(9: 11, v / v)$ at room temperature for one week (m.p. $140-142^{\circ} \mathrm{C}$ ).

\section{Experimental details}

$\mathrm{H}$ atoms were placed in calculated positions with $d(\mathrm{C}-\mathrm{H})=0.93$ (aromatic), $0.97 \AA$ (methylene) and $d(\mathrm{~N}-\mathrm{H})=0.86 \AA$, and were refined in riding mode with $U_{\text {iso }}(\mathrm{H})=1.2 U_{\mathrm{eq}}(\mathrm{C}, \mathrm{N})$.

\section{Discussion}

In the title crystal structure, the conformations of the $\mathrm{N}-\mathrm{O}$ and $\mathrm{C}=\mathrm{O}$ bonds are anti to each other (figure, top), similar to that observed in 1-(2-fluorobenzyl)-1-(2-fluorobenzyloxy)urea [1], $\mathrm{N}$ hydroxyurea [2-5], 1-hydroxy-1-methylurea and 1-hydroxy-3methylurea [6]. The length of the carbonyl bond $(C=0)$ in above hydroxyurea derivates [2-6] is longer (> 1.25 $\AA$ ), but the values observed in the title compound is in the normal range of 1.19 $1.23 \AA$ and obviously shorter $(<1.25 \AA)$. This may be related with the hydroxyl group etherification, which suggests that the structure has not an $\mathrm{H}$ atom of hydroxyl group to form intramolecular hydrogen bonding with 02 . The $\mathrm{C}-\mathrm{N}$ bond lengths in the urea group $(1.274(3)-1.326(3) \AA$, mean $1.300(3) \AA$ ) are longer than a typical $\mathrm{C}=\mathrm{N}$ double bond (mean $1.269 \AA$ ), but shorter than a $\mathrm{C}-\mathrm{N}$ single bond (mean 1.443(4) $\AA$ ), indicating electron delocalization in the urea group [7]. The group $\mathrm{N}-(\mathrm{C}=\mathrm{O})-\mathrm{N}$ urea planar forms a dihedral angle of $71.5(2)^{\circ}$ with the benzyl group, and the $\mathrm{N}-\mathrm{O}$ bonds are twisted by about $20^{\circ}$ out of the $\mathrm{N}-(\mathrm{C}=\mathrm{O})-\mathrm{N}$ urea planes. In the crystal structure, molecules are linked into antiparallel chains running along [010] by intermolecular $\mathrm{N}-\mathrm{H}$... O hydrogen bonding (figure, bottom).

Table 1. Data collection and handling.

\section{Crystal:}

Wavelength:

$\mu$ :

Diffractometer, scan mode:

$2 \theta_{\max }$ :

$N(h k l)_{\text {measured, }} N(h k l)_{\text {unique: }}$

Criterion for $I_{\mathrm{obs}}, N(h k l) \mathrm{g}$ :

$N(\text { param })_{\text {refined: }}$

Programs:

Table 2. Atomic coordinates and displacement parameters (in $\AA^{2}$ ).

\begin{tabular}{llllll}
\hline Atom & Site & $x$ & $y$ & $z$ & $U_{\text {iso }}$ \\
\hline H(1A) & $4 e$ & 0.2376 & 1.0445 & 1.0679 & 0.076 \\
H(1B) & $4 e$ & 0.2503 & 1.2966 & 1.0009 & 0.076 \\
H(7) & $4 e$ & 0.1055 & 0.7731 & 1.0108 & 0.115 \\
H(6) & $4 e$ & 0.0182 & 0.5614 & 0.8809 & 0.150 \\
H(3) & $4 e$ & 0.2954 & 1.1400 & 0.8215 & 0.102 \\
H(5) & $4 e$ & 0.0662 & 0.6487 & 0.7254 & 0.113 \\
H(4) & $4 e$ & 0.2074 & 0.9271 & 0.6922 & 0.112 \\
H(1) & $4 e$ & 0.3680 & 0.6409 & 0.9926 & 0.063 \\
H(2A) & $4 e$ & 0.4599 & 0.9426 & 1.2365 & 0.066 \\
H(2B) & $4 e$ & 0.4050 & 1.1021 & 1.1539 & 0.066 \\
\hline
\end{tabular}

\footnotetext{
* Correspondence author (e-mail: cmxlf2008@163.com)
} 
Table 3. Atomic coordinates and displacement parameters (in $\AA^{2}$ ).

\begin{tabular}{|c|c|c|c|c|c|c|c|c|c|c|}
\hline Atom & Site & $x$ & $y$ & $z$ & $U_{11}$ & $U_{22}$ & $U_{33}$ & $U_{12}$ & $U_{13}$ & $U_{23}$ \\
\hline$C(1)$ & $4 e$ & $0.2619(2)$ & $1.1055(6)$ & $1.0066(2)$ & $0.075(2)$ & $0.051(2)$ & $0.061(2)$ & $0.011(1)$ & $-0.017(1)$ & $-0.003(1)$ \\
\hline$C(2)$ & $4 e$ & $0.2072(2)$ & $0.9724(5)$ & $0.9271(2)$ & $0.056(1)$ & $0.050(2)$ & $0.056(1)$ & $0.009(1)$ & $-0.015(1)$ & $0.004(1)$ \\
\hline$C(7)$ & $4 e$ & $0.1237(3)$ & $0.7997(8)$ & $0.9473(2)$ & $0.102(2)$ & $0.110(3)$ & $0.071(2)$ & $-0.038(2)$ & $-0.024(2)$ & $0.026(2)$ \\
\hline$C(6)$ & $4 e$ & $0.0739(4)$ & $0.681(1)$ & $0.8728(3)$ & $0.121(3)$ & $0.131(4)$ & $0.109(3)$ & $-0.068(3)$ & $-0.047(2)$ & $0.029(3)$ \\
\hline$C(3)$ & $4 e$ & $0.2393(3)$ & $1.0227(8)$ & $0.8299(2)$ & $0.073(2)$ & $0.112(3)$ & $0.066(2)$ & $-0.022(2)$ & $-0.012(2)$ & $0.003(2)$ \\
\hline$C(5)$ & $4 e$ & $0.1052(3)$ & $0.7341(8)$ & $0.7781(3)$ & $0.090(2)$ & $0.095(3)$ & $0.088(2)$ & $-0.006(2)$ & $-0.041(2)$ & $-0.004(2)$ \\
\hline$C(4)$ & $4 e$ & $0.1894(3)$ & $0.9035(9)$ & $0.7559(2)$ & $0.088(2)$ & $0.131(3)$ & $0.057(2)$ & $0.004(2)$ & $-0.016(2)$ & $-0.003(2)$ \\
\hline$O(1)$ & $4 e$ & $0.3742(1)$ & $1.0419(3)$ & $1.0028(1)$ & $0.068(1)$ & $0.0375(9)$ & $0.055(1)$ & $-0.0016(7)$ & $-0.0258(8)$ & $0.0075(7)$ \\
\hline $\mathbf{N}(1)$ & $4 e$ & $0.3887(2)$ & $0.7727(4)$ & $1.0303(1)$ & $0.073(1)$ & $0.030(1)$ & $0.047(1)$ & $-0.0045(8)$ & $-0.0257(9)$ & $0.0001(8)$ \\
\hline$C(8)$ & $4 e$ & $0.4358(2)$ & $0.7452(4)$ & $1.1169(2)$ & $0.063(1)$ & $0.031(1)$ & $0.040(1)$ & $-0.0040(9)$ & $-0.0117(9)$ & $0.0008(9)$ \\
\hline$O(2)$ & $4 e$ & $0.4724(2)$ & $0.5232(3)$ & $1.1362(1)$ & $0.093(1)$ & $0.0291(9)$ & $0.0467(9)$ & $0.0027(8)$ & $-0.0262(8)$ & $-0.0001(6)$ \\
\hline $\mathbf{N}(2)$ & $4 e$ & $0.4333(2)$ & $0.9545(4)$ & $1.1760(1)$ & $0.083(1)$ & $0.033(1)$ & $0.044(1)$ & $0.0042(9)$ & $-0.0213(9)$ & $-0.0033(8)$ \\
\hline
\end{tabular}

Acknowledgments. The authors gratefully acknowledge the financial support of this study by National Key S\&T Special Project of China: Grand New Drug R\&D (grant no. 2009ZX09103-087) and the support of Grand Science and Technology Special Project of Jiangxi Province,China (grant no. 20041A0300201). The authors aiso thank JingGangShan College and Professor Xiao-Niu Fang for assistance with the date collections and refinements.

\section{References}

1. Armagan, N.; Richards, J. P. G.; Uraz, A. A.: The Structure of Hydroxyurea at Liquid-Nitrogen Temperature. Acta Crystallogr. B32 (1976) 1042.

2. Berman, H.; Kim, S. H.: A second determination of the structure of hydroxyurea. Acta Crystallogr. 23 (1967) 180.

3. Bettina, B. N.; Karla, F.; Ingrid, K. L.: Structures of Two Ribonucleotide Reductase Inhibitors: 1-Hydroxy-1-methylurea and 1-Hydroxy-3methylurea. Acta Crystallogr. C49 (1993) 1018-1022.

4. Ding, Q. C.; Huang, Y. L.; Jin, J. Y.; Zhang, L. X.; Fu, X. D.; Cai, X. Q.: Crystal structure of 3-(2,4-dinitrophenylthio)-5-(2-ethoxyphenyl)-4H1,2,4-triazol-4-amine, $\mathrm{C}_{16} \mathrm{H}_{14} \mathrm{~N}_{6} \mathrm{O}_{5} S$. Z. Kristallogr. NCS 224 (2009) 103-104.

5. Ingrid, K. L.; Bodil, J.: The Crystal and Molecular Structure of Hydroxyurea. Acta Chem. Scand. 20 (1966) 983-991.
6. Mai, X.; Xia, H. Y.; Cao, Y.S.; Lu, X.S.; Fang, X. N.: 1-(2-fluorobenzyl)1-(2-fluorobenzyloxy)urea. Acta Crystallogr. E65 (2009) 0442.

7. Thiessen, W. E.; Levy, H. A.; Flaig, B. D.: Non-Planarity of Hydroxamic Acids. Structures of 3-Hydroxyxanthine Dihydrate by X-ray Diffraction and Hydroxyurea by Neutron Diffraction. Acta Crystallogr. B34 (1978) 2495.

8. Sheldrick, G. M.: SHELXS-97. Program for the Solution of Crystal Structures. University of Göttingen, Germany 1997.

9. Sheldrick, G. M.: SHELXL-97. Program for the Refinement of Crystal Structures. University of Göttingen, Germany 1997. 